\title{
XD: A Cross-Layer Designed Data Collection Mechanism for Mission-Critical WSNs in Urban Buildings
}

\author{
Chieh-Ting Huang ${ }^{a}$, Tsung-Han Lin ${ }^{b}$, Ling-Jyh Chen ${ }^{c}$, Polly Huang ${ }^{a}$ \\ ${ }^{a}$ Department of Electrical Engineering \\ National Taiwan University \\ ${ }^{b}$ School of Engineering and Applied Sciences \\ Harvard University \\ ${ }^{c}$ Institute of Information Science \\ Academia Sinica \\ r96921024@ntu.edu.tw, thlin@eecs.harvard.edu, cclljj@iis.sinica.edu.tw, phuang@cc.ee.ntu.edu.tw
}

\begin{abstract}
As the R\&D experience accumulates, there is a rising interest of wireless sensor network (WSN) deployment in the urban environment. For mission critical applications such as healthcare and workplace safety, in particular, it is essential that the data dissemination mechanisms satisfy two important quality of service (QoS) requirements: (1) high delivery rate and (2) low transmission delay. Proposed in this work is a cross-layer designed data dissemination mechanism, referred to as Cross-Layer Diffusion (XD), in which notions in the path discovery (routing) component are exploited by data forwarding (MAC) component to improve the delivery rate and transmission delay. Using traces collected from a prototype WSN deployed in urban environment, we compare XD to the state-of-the-art mechanisms and find that XD is not only more efficient but also more practical.
\end{abstract}

Keywords- Urban Wireless Sensor Network; Data Dissemination, Cross-Layer Design

\section{INTRODUCTION}

Envisioning a new generation of mission-critical applications in the urban environment, we seek mechanisms that provide reliable and timely transmissions of sensor data. The two QoS requirements, (1) high data delivery rate and (2) low end-to-end transmission delay, need to be jointly considered when devising such mechanisms. It is, however, not trivial to satisfy the two requirements at the same time. For high delivery rate, one often takes the ARQ approach [1] to retransmit lost data. This adds to the end-to-end delay and, in a way, trades off the other QoS requirement for missioncritical applications. Alternatively, one may take the FEC approach [2] which transmits redundant data to raise the probability of having at least one copy arriving at the data sink. This approach appears to allow timely delivery of data in the presence of loss, but it could also come back to haunt the network in terms of both loss and delay if the degree of redundancy is not cautiously administered. Taking an FECwith-care approach, we propose a cross-layer designed data collection mechanism, referred to as Cross-Layer Diffusion (XD).

$\mathrm{XD}$ is a mission-critical data collection mechanism with (1) opportunistic redundancy and (2) collision avoidance. The path discovery part of XD is based on a multi-path shortest path routing mechanism referred to as Magnetic Diffusion (MD) [3]. In that, the network establishes a magnetic field across the WSN, with decreasing magnetic charges radiating from the sink. Sensor data, in turn, travel the network tending to the nodes with higher magnetic charges until reaching the sink, mimicking how metallic objects (data) are attracted by a magnet (sink). If there exist multiple shortest paths in the WSN topology, all of such paths will be discovered by the mechanism. As a result, sensor data are disseminated with redundancy in a way that duplicates will travel multiple shortest paths. Such redundancy is desired by mission-critical applications in that the data delivery rate tends to be high and the end-to-end delay tends to be low. Because the degree of redundancy depends on the network topology, we refer to this property of $\mathrm{XD}$ as opportunistic redundancy.

The data forwarding part of XD is co-designed with the path discovery component. In that, a hybrid TDMA and CSMA mechanism is proposed to administer the forwarding of sensor data. The rationale behind such a design is that, with the opportunistic redundancy, there will be a higher amount of bits travelling the WSN which increases the chance of collision. TDMA-based solutions are effective avoiding collisions in high traffic load cases. However, conventional TDMA divides the time slots in a cycle by individual nodes in the neighborhood. This results in higher end-to-end delays in densely-deployed WSNs. The conventional TDMA mechanisms do not scale to the density of the WSNs.

Our contribution lies in the design of a novel TDMA mechanism utilizing the magnetic charges established by the path discovery component. In our design, nodes of the same magnetic charge send data at the same time slot. A cycle is divided into three time slots only. A node with magnetic charge $\mathrm{C}$ transmits data at time slot $\mathrm{C} \bmod 3$. This prevents data coming from a level up or a level down to collide at the middle level (hidden terminal). Furthermore, to avoid data collisions among the nodes of the same charge, a CSMA mechanism is applied within each level. I.e., each node listens to the channel and makes sure other nodes at the same charge level are indeed idle before transmitting. The TDMA 
and CSMA combined contribute to the collision avoidance property of XD.

This simple, cross-layer designed mechanism is implemented in ns-2 [4]. Using traces collected from an WSN deployed in an urban building to drive the simulations, we compare XD to two other protocol stacks, MD with ZMAC [8] and Zigbee [5] in terms of data delivery rate and end-to-end delay. The former represents the use of the multipath discovery mechanism with a state-of-the-art TDMA MAC protocol and the latter represents the commonly-used industrial standard. The simulation results indicate that XD performs significantly better than the Zigbee stack. Additionally, XD performs comparably well to the state-ofthe-art while the complexity of establishing the TDMA schedule is significantly lower. These evidences support that $\mathrm{XD}$ is an effective and practical data collection mechanism for mission-critical WSNs.

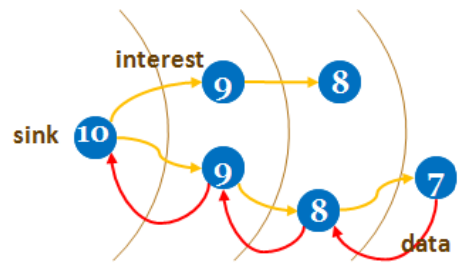

Figure 1. Flow of Control and Data Packets in MD

\section{MECHANISM}

Cross-layer Diffusion (XD) is simple and yet effective. The path discovery component implements a multi-path shortest path routing mechanism. The data forwarding component implements a hybrid CSMA and TDMA mechanism based on the notion in the path discovery component. In this section, we describe the two components of XD in detail and provide the rationale of how the crosslayer designed mechanism achieves reliable and timely sensor data collection.

\section{A. Path Discovery}

The design of MD is inspired by the physics between magnets and metallic objects. Consider the data sink as a magnet and the data as metallic nails. The data will be attracted towards the sink according to the magnetic field just as the nails are attracted towards the magnet. The magnetic field is established by setting up the proper magnetic charges on the sensor nodes within the magnetic influence of the data sink. The strength of the charge is determined by the hop distance to the sink. The data will be propagated based on the magnetic field from low-charge to high-charge nodes. By this principle, data traveling towards the center of attraction, the sensor nodes forward data coming strictly from the nodes with smaller charges. Forwarding data this way, the paths selected by MD are the shortest in hop count. Furthermore, there can be multiple next hops to forward the data. Thus, MD sends data in an optimal multi-path fashion.

MD operates in two phases: the interest broadcast and data propagation. Figure 1 shows the flow of interest and data packets in MD. The number on each node indicates the magnetic charge assigned. The magnetic field is established in the interest broadcast phase such that the data can be disseminated towards the sink in the data propagation phase. At the interest broadcast phase, the sink whose magnetic charge is set the highest charge and broadcasts the interest packet to its neighbors. When a node receives an interest message, it decreases the magnetic charge in the interest by one. The node then records this magnetic charge and forward the interest message to its neighbors. The decrementing magnetic charges from the sink to source will guide flow of data in the reverse direction, from high-charge to low-charge points in the magnetic field.

The second phase, data dissemination, a node senses the data and broadcasts packets with the charge of itself. Therefore, the receiving node can identify from the charge carried in the data where the data is coming from and whether to forward the data further down the stream. The node compares the magnetic charge of the data with the charge of itself. If the magnetic charge in the data smaller, the node increases the magnetic charge of the data by one and continues to disseminate the data. On the other hand, if a node receives a duplicate data or the data whose magnetic charge is smaller than the node's, the data will be discarded.

\section{B. Data Forwarding}

XD incorporates a hybrid TDMA-CSMA MAC mechanism that utilizes information established by MD. Even if there is no contention in the network, the data delivery rate may still suffer from losses due to bit errors commonly seen in wireless networks. Our design objective
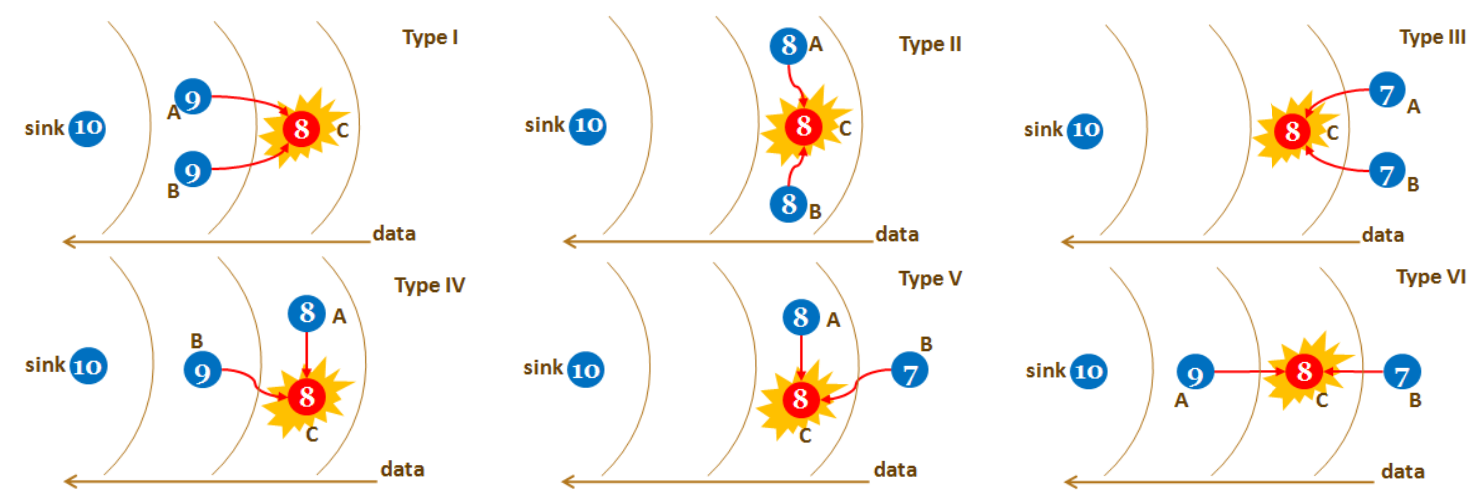

Figure 2. Types of Collision in MD 


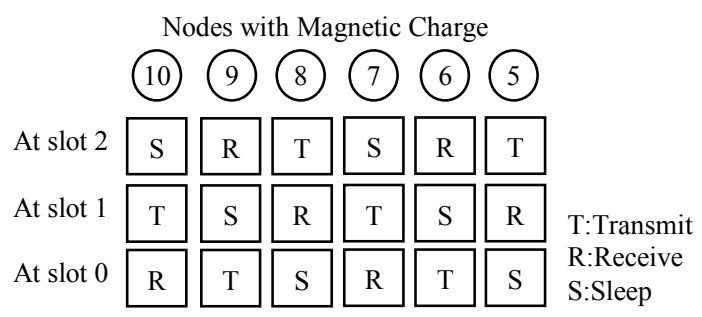

Figure 3. Three-Level TDMA Schedule

is thus not to establish a $100 \%$ contention-free network but to avoid as many collisions as possible with redundancy. More specifically, the TDMA part of the design eliminates collisions from hidden terminals two magnetic charge levels apart and its CSMA counterpart avoids collisions between neighboring nodes.

To elaborate in more detail the design rationale, we classify collisions in MD networks into six canonical types. In Figure 2, node A and B are sending nodes and the packets collide at node $\mathrm{C}$. The number on the node indicates the assigned magnetic charge. Type I, II and III are cases where sending nodes are at the same level. In that, the sending nodes can be one level higher, at the same level, or one level lower than the receiving node. Type I and II collisions are not critical because these data are traveling against the magnetic field. Type III collisions are, however, problematic. These collisions can be reduced partly by applying CSMA at nodes within the same level since nodes at the same level tend to be nearby. Note that, though, there remains a slight chance where nodes at the same level are away from each other's carrier sense range.

Type IV and V are cases where sending nodes are of adjacent magnetic charges. Again, Type IV is not critical given the transmission is not effective for data collection at the sink. Type V collisions, the critical ones, can be reduced by CSMA to a certain degree because nodes with adjacent magnetic charges can hear each other relatively easily. A significant amount of the collisions can be further reduced by separating the sending time of the nodes at different levels, hinting the benefit of a TDMA strategy to the design. In Type VI, where the two sending nodes are two levels apart, nodes of higher and smaller level become hidden terminals to each other. These collisions may not be eliminated by carrier sense. The sending time of nodes two levels apart needs to be separated to avoid collisions effectively. To avoid type V and VI collisions effectively, TDMA based on the magnetic charge levels is applied.

$\mathrm{XD}$ uses three time slots for the TDMA schedule. The slot assignment is based on each node's magnetic charge. In the interest broadcast phase of $\mathrm{XD}$, the interest messages,

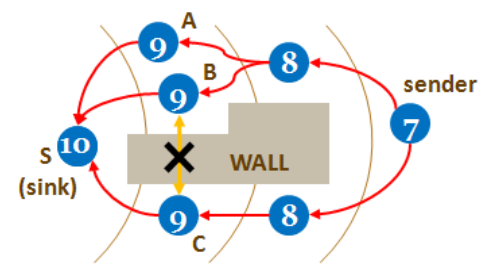

Figure 5. Hidden Terminal Effect around Sink.

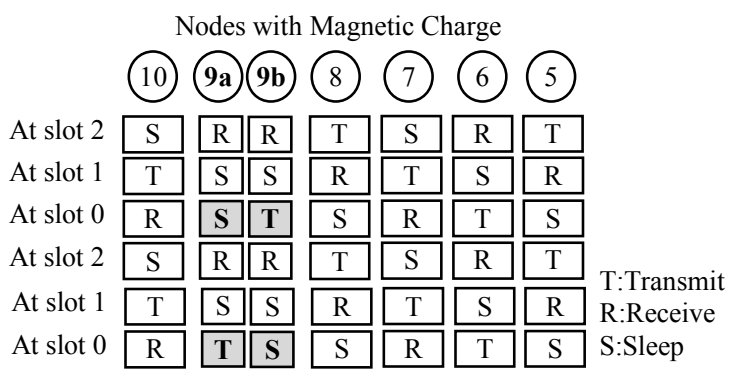

Figure 4. Extended TDMA Schedule

originated from the sink, carry magnetic charges decremented at each hop. When a node receives the interest message, the node records the magnetic charge in the message and the TDMA slot for the node is assigned to $\mathrm{n}$ $\bmod 3$, where $\mathrm{n}$ is the recorded magnetic charge.

An example of the TDMA mechanism is shown in Figure 3 . The numbers in the circles are the magnetic charges after the interest broadcast phase. In slot two, only nodes with charge 8 and 5 can send packets and the packets can be received by the nodes that are downstream, e.g. nodes with charge 9 and 6 respectively. At this time, nodes with charge 10 and 7 will go into the sleep mode since receiving packets from their downstream neighbors is not necessary. In the next time slot, those nodes, who receive packets from upstream nodes at previous slot, will go into the transmit mode and transmit the packets to the nodes at the next level.

Furthermore, XD may discover multiple paths to deliver data. Packets travelling multiple disjoint paths may collide at the sink node. Consider an urban WSN shown in Figure 5. The sender sends packets to the sink via two disjoint paths. Though node A, B and C are neighbors of the sink, node $\mathrm{C}$ is separated from node $\mathrm{A}$ and $\mathrm{B}$ by a corner in an indoor environment. In this case, $\mathrm{A}$ and $\mathrm{B}$ will not send data simultaneously due to CSMA. However, node B and C cannot hear each other thus collisions may still occur when node $\mathrm{C}$ also sends packets. In order to prevent data collisions at the sink node, XD further refines the TDMA schedule at the sink node. The sink node's neighbors are grouped. Each group shares the same carrier sense region. By collecting neighbors' information at the sink, the sink can determine how many groups and the appropriate schedule separating the sending of data from each group. Each time slot is divided into multiple sub-slots depending on the number of groups there are. An example of the refined schedule is shown in Figure 4. The nodes with charge 9 are the sink neighbors. That slot is further divided into two for two groups of neighbors, i.e., $9 \mathrm{a}$ and $9 \mathrm{~b}$. When $1 \mathrm{a}$ transmits packets, $9 \mathrm{~b}$ goes to sleep. When $9 \mathrm{a}$ goes to sleep, $9 \mathrm{~b}$ transmits packets.

\section{TRACE-DRIVEN SIMULATION}

To examine the performance of XD, we conduct a set of trace-driven simulations using ns-2 [4]. This methodology enables us to (1) repeat the tests, (2) reuse the existing simulation implementation, and in the meantime (3) capture the effect of packet losses occurring in realistic urban buildings. 


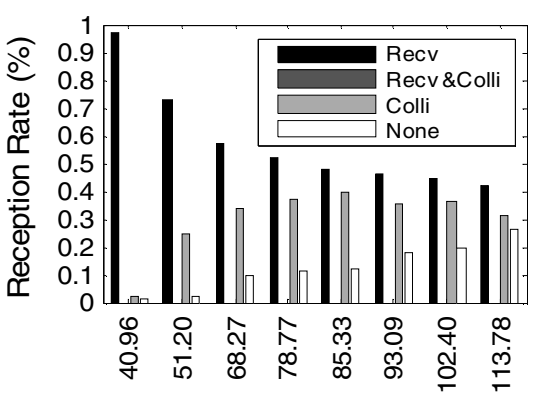

first-hop node

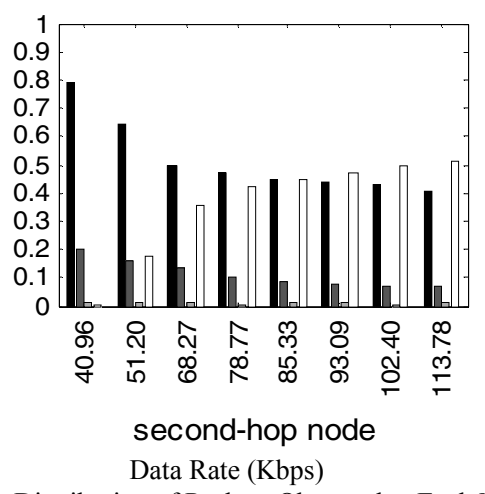

Data Rate (Kbps)

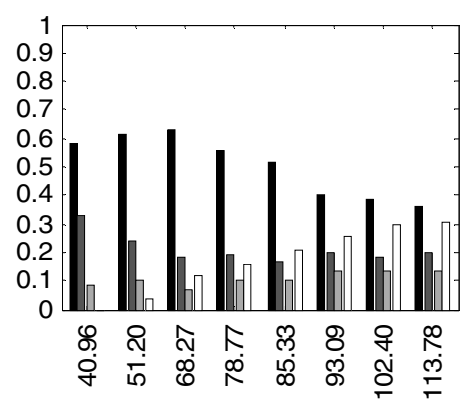

sink node

Figure 8. Distribution of Packets Observed at Each Node.

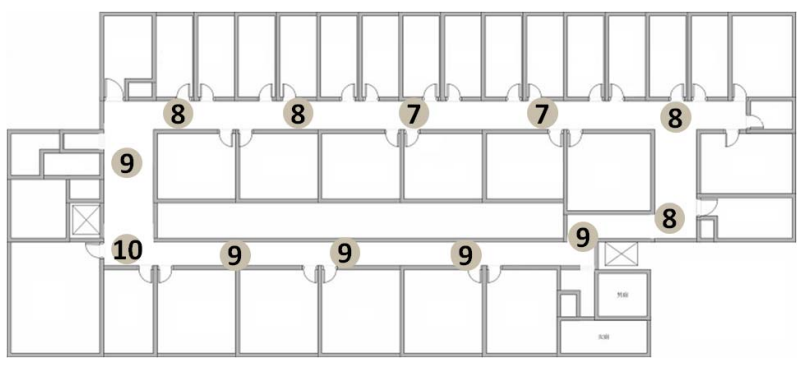

Figure 6. Testbed Topology.

Our implementation of XD includes (1) MD and (2) the hybrid MAC. The magnetic charges set by the MD component are communicated to the MAC component for the magnetic-level-based TDMA scheduling. $\mathrm{XD}$ is compared to two suites to data collection protocols. One is the well-known Zigbee stack. The MAC component of Zigbee is a simplified version of the native IEEE 802.15.4 module [6] in ns-2, and the routing component is adopted from the AODV module [7] in ns-2. The other suite consists of MD and a state-of-the-art MAC, ZMAC [8]. One unique feature in our simulation approach is the use of packet loss patterns collected from an actual WSN. Instead of the builtin wireless link propagation models implemented in ns-2, we implemented a trace-driven physical layer module which allows us to simulate realistic wireless packet losses and collisions in an indoor environment.

Figure 6 illustrates the actual WSN used to collect the traces for the physical layer module. The testbed is deployed on the $6^{\text {th }}$ floor of the Electrical Engineering Department building of NTU.. There are 12 nodes in the testbed. We measure the packet reception rate (PRR) of each node by programming 11 nodes as receiver and 1 node as sender. Each of the 12 nodes will take turn to be the sender. The PRR is obtained by calculating the ratio of the number of packets received to the number of packets sent. In the simulation, when a node sends a packet, other nodes determines based on the measured PRR whether the packet can be heard at the intended receiver. Based on the preliminary measurement results, we observe that the indoor wireless links are often asymmetric, very distinctive of the propagation models, which are more appropriate for open space transmissions, implemented in ns- 2 .
The exact topology of the testbed is used to drive the ns-2 simulations as well. The bottom left node in Figure 6 is the data sink. The data sink's charge is set to 10 . The magnetic charge after the interest broadcast phase of MD for each node is shown as the number in the node. There are two nodes of the lowest charge, 7 . The one on the left is the data source. In each simulation, the source sends 1000 packets before the simulation terminates.

\section{Performance AnAlysis}

We first analyze the effect of implementing the TDMA mechanism around the sink node and the TDMA mechanism based on the magnetic charges. Figure 7 shows the CDF of end-to-end delay in MD with simple CSMA. In this set of simulations, we control all factors but the sending rate of the source node, from $22.76 \mathrm{kbps}$ to $113.78 \mathrm{kbps}$. We can observe that the delay increases and reception rate decreases as the source data rate increases. In particular, (1) when data rate is below $68.27 \mathrm{kbps}$, the end-to-end delay is generally low, but (2) the data delivery rate is only about $80-90 \%$ at best. This is to our surprise knowing that the capacity of IEEE 802.15 .4 is $250 \mathrm{kbps}$ at the physical level. The utilization of the $22.76 \mathrm{kbps}$ workload case is only about $20 \%$ (two charge-8 nodes help forwarding).

To understand the reason of such a low data delivery rate when the workload is low, we classify packets sent from the source into four types by how they are received at each node along the transmission path. Because XD allows packets to travel multiple paths, it is often a node receives duplicates of individual packets. If all duplicates of a packet are successfully received at the node, the packet is categorized as

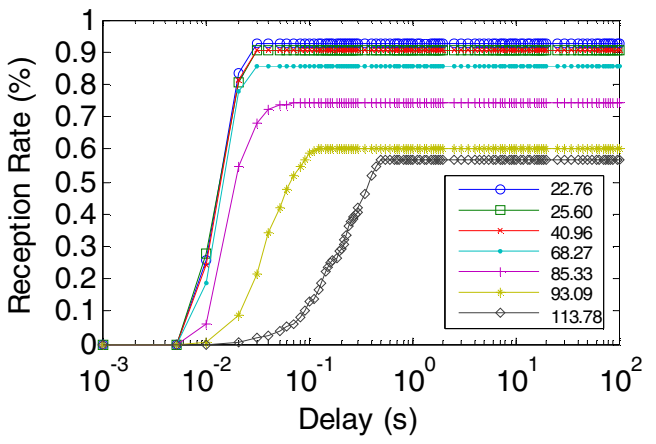

Figure 7. CDF of End-to-End Delay in MD with CSMA. 


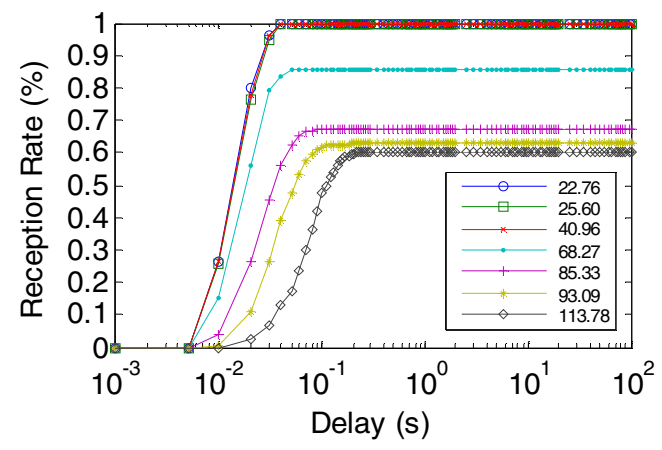

Figure 9. CDF of End-to-End Delay in MD with TDMA around Sink.

the first type (Recv). If a certain duplicate of a packet arrives while at least one of the duplicates is collided, the packet is categorized into the second type (Recv \& Colli). The third type is for packets whose duplicates are all collided (Colli). The last type is for packets that are never sent by the upstream nodes (None). These are the packets that might have been collided at upstream nodes. The sum of the four types is $100 \%$ which is the total number of packets sent by the data source.

The three plots in Figure 8 depict the portion of the packets of each type received at nodes from the first-hop neighbor of the source towards the sink. Each column represents the portion of the packets of each type at different sending rate. One can observe that, at the first-hop node, there are an increasing number of packets being collided as the workload increases. Most of the packets are collided due to the hidden terminal effect between the source and the second-hop nodes. The portion of packets that are never seen at the first hop node also increases as the sending queue at the source node builds up faster and the packets are eventually dropped at the source node before they get to travel the network at all. The packets collided at the first hop and the packets never sent from the source will not reach the second-hop node at all. The amount is indicated as the type four packets (None) in the middle plot. There is a minor amount of collisions at the second-hop nodes which results in small portion of type two and type three packets. This is because the sink node does not forward data further. Therefore, there is no observation of collisions due to hidden terminals between the sink and the first-hop node at the second-hop node.

The behavior at the sink node, i.e., the third-hop node, is particularly interesting. One would expect the portion of type four packets to be at least the amount observed in the second-hop node. The simulation results reflect the property of multi-path routing where packets might travel through other paths to reach the data sink. In this case, a significant amount of data eventually reaches the data sink. Also due to the fact that packets may travel multiple paths in the network, there is a slightly higher amount of collisions observed at the sink node. These contribute to the higher amounts of type two and type three packets observed at the sink node.

The performance of multi-path routing with simple CSMA is rather interesting in that (1) the multiple paths

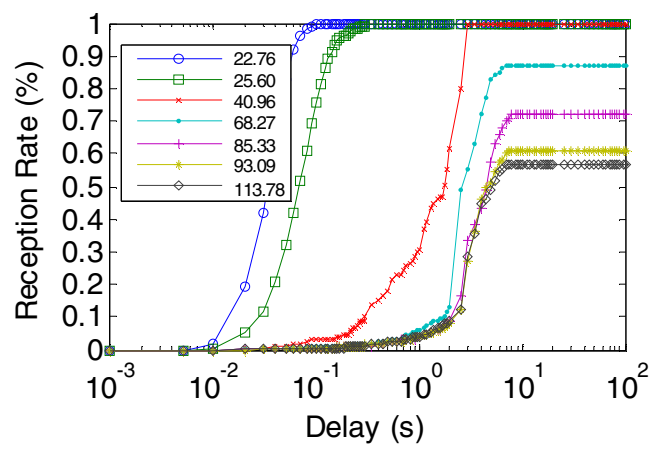

Figure 10. CDF of End-to-End Delay in XD

result in a higher delivery rate at the sink when the workload is high but in the meantime (2) the hidden terminal effect around the sink node also results in collisions which lowers the data delivery rate when the workload is low. We then turn on the TDMA around the sink node to eliminate the hidden terminal effects observed above. Figure 9 shows the CDF of end-to-end delay in MD with CSMA and TDMA around sink. The data delivery rate at the low traffic load case is effectively raised to $100 \%$ while the end-to-end delay is slightly compromised.

Figure 10 shows the CDF of end-to-end delay of complete XD. Because XD schedules both sink neighbors and nodes from different magnetic charges, it eliminates both hidden terminal problem at the sink node and the intermediate nodes (the first-hop and second-hop nodes). Important performance properties observed are as follows. (1) The data delivery rate in low-load cases are almost $100 \%$. (2) In mid-load cases, e.g., $68.27 \mathrm{kbps}$ and $85.33 \mathrm{kbps}$, XD helps raising the data delivery rate. This, however, trades off the end-to-end delay, a problem general to TDMA-based solutions. Packets need to wait in the queue for the time slots assigned and such waiting repeats at each node along the transmission path. The queuing delay accumulates. (3) When the data rate is high, i.e., the traffic is close to the network capacity, the queue may overflow and result in packet drops, a problem that can only be alleviated by extending the network capacity.

\section{COMPARISON}

We next compare XD to two other protocol stacks for sensor data collection. The first stack is Zigbee, using AODV for path discovery and IEEE802.15.4 for data forwarding. The other stack uses MD for path discovery and ZMAC for data forwarding.

\section{A. AODV with IEEE802.15.4:}

AODV is an on-demand single-path routing protocol. When a node needs to transmit data, AODV lookups its routing table to see is there exists a routing path to the destination. If not, the packet is buffered at the AODV queue (not the queue at MAC layer) while the mechanism begins to discover a routing path. This introduces a certain amount of delay. 


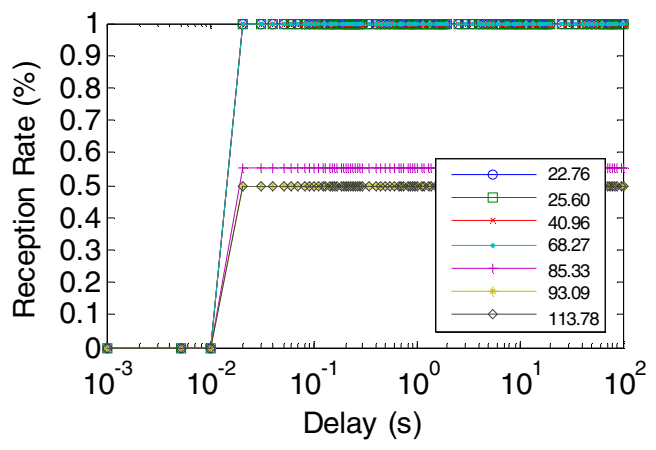

Figure 11. CDF of End-to-End Delay in Zigbee Stack.

The MAC mechanism recommended in IEEE802.15.4 is CSMA-like. The end-to-end delay of packets, if received at the sink, is expected small. Figure 11 shows the CDF of endto-end delay. The delay is indeed low. The data delivery rate is close $100 \%$ when the workload is low. However, when traffic load increases, the collisions due to hidden terminal problem along the transmission path occur. About half of the packets are collided which results in a significant data delivery rate for mid-load and high-load cases.

\section{B. MD with ZMAC:}

ZMAC is a hybrid CSMA and TDMA mechanism. The schedule used by its TDMA component is derived by the DRAND [9] algorithm which requires topological information in the two-hop neighborhood. Each node exchanges information about its two-hop neighbors and then calculates a proper TDMA slot for itself. This is how ZMAC presents hidden terminals along the transmission paths from occurring. In ZMAC, although every node has its slots for transmission, it allows nodes competing sending data at the slots which are not assigned. The CDF of end-to-end delay is shown in Figure 12. When data rate is high, packets travelling the network need to wait longer in the queue and the nodes start to drop packets when the queue is full. Similar to XD, the data delivery rate is close to $100 \%$ in lowload cases. From mid-load cases and on, the end-to-end delay is raised to the scale of seconds and the data delivery rate begins to drop afterwards.

In summary, there is no significant difference in terms of performance in extreme low- and high-load cases. However, in mid-load cases, although Zigbee can achieve low transmission delay, it is significantly less efficient in data delivery rate. MD with ZMAC shows comparable performance as XD. It is only slightly worse in terms of data delivery rate in the mid-load cases.

The complexity of DRAND, the algorithm for establishing the TDMA schedule in ZMAC, is substantial. For WSNs with mobile nodes in the topology, the schedule needs to be recomputed repeatedly. As in XD, the schedule is determined by the magnetic charge and is set by the interest message propagated periodically, XD updates its TDMA schedule in the meantime it rediscovers paths. Relative to the MD-ZMAC combination, XD can be applied to a wider range of mission-critical WSNs in urban environments.

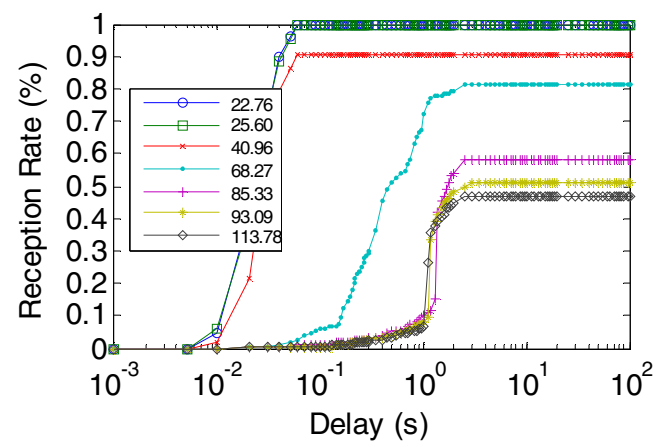

Figure 12. CDF of End-to-End Delay in MD+ZMAC Stack.

\section{CONCLUSION}

We present in this work $\mathrm{XD}$, a cross-layer designed sensor data collection mechanism. Through a set of tracedriven simulations, we find that by scheduling sink neighbors and nodes according to magnetic charge, XD prevents most collisions due to the hidden terminal effect. Combined with the multiple shortest paths discovered by $\mathrm{MD}, \mathrm{XD}$ performs, in terms of data delivery rate and end-toend delay, better than the Zigbee stack and just as well to the state of the art. Given the simplicity of TDMA schedule establishment algorithm in XD, it is not just efficient but also practical for mission-critical WSNs in urban buildings.

\section{REFERENCES}

[1] S. Wicker, Error Control Systems for Digital Communications and Storage. Englewood Cliffs, NJ: Prentice Hall, Inc., 1995.

[2] S. Lin, and D. J. Costello, Jr., Error control coding: fundamentals and applications, Prentice-Hall, 1983.

[3] Hsing-Jung Huang, Ting-Hao Chang, Shu-Yu Hu and Polly Huang, "Magnetic Diffusion: Scalability, Reliability, and QoS of Data Dissemination Mechanisms for Wireless Sensor Networks," Computer Communications, Vol. 29, No. 13, pp. 2482-2493, Aug. 2006

[4] Lee Breslau, Deborah Estrin, Kevin Fall, Sally Floyd, John Heidemann, Ahmed Helmy, Polly Huang, Steven McCanne, Kannan Varadhan, Ya Xu and Haobo Yu, "Advances in Network Simulation," The VINT Project IEEE Computer, Vol. 33, No. 5, pp. 59-67, May 2000

[5] ZigBee Alliance, ZigBee Specifications, version 1.0, April 2005

[6] J. Zheng and Myung J. Lee, "A comprehensive performance study of IEEE 802.15.4," Sensor Network Operations, IEEE Press, Wiley Interscience, Chapter 4, pp. 218-237, 2006.

[7] J. Broch, D. Maltz, D. Johnson, Y. Hu and J. Jetcheva, "A Performance Comparison of Multi-Hop Wireless Ad Hoc Network Routing Protocols," In the Proceedings of the Fourth Annual ACM/IEEE International Conference on Mobile Computing and Networking (MobiCom 2008), 2008

[8] Injong Rhee, Ajit Warrier, Mahesh Aia and Jeongki Min, "ZMAC: a Hybrid MAC for Wireless Sensor Networks," IEEE/ACM Transactions on Networking, June 2008

[9] I. Rhee, A. Warrier, and L. Xu, "Randomized dining philosophers to TDMA scheduling in wireless sensor networks," Technical report, Computer Science Department, North Carolina State University, Raleigh, NC, 2004. 